\title{
Gestaltungsorientierte Forschung am Beispiel der Referenzmodellierung in der Telekommunikationsindustrie
}

\author{
Christian Czarnecki · Christian Dietze
}

Eingegangen: 30. Oktober 2019 / Angenommen: 30. Januar 2020 / Online publiziert: 10. Februar 2020

(C) Der/die Autor(en) 2020

Zusammenfassung Am Beispiel der Telekommunikationsindustrie zeigt der Beitrag eine konkrete Ausgestaltung anwendungsorientierter Forschung, die sowohl für die Praxis als auch für die Wissenschaft nutzen- und erkenntnisbringend ist. Forschungsgegenstand sind die Referenzmodelle des Industriegremiums TM Forum, die von vielen Telekommunikationsunternehmen zur Transformation ihrer Strukturen und Systeme genutzt werden. Es wird die langjährige Forschungstätigkeit bei der Weiterentwicklung und Anwendung dieser Referenzmodelle beschrieben. Dabei wird ein konsequent gestaltungsorientierter Forschungsansatz verfolgt. Das Zusammenspiel aus kontinuierlicher Weiterentwicklung in Zusammenarbeit mit einem Industriegremium und der Anwendung in vielfältigen Praxisprojekten führt zu einer erfolgreichen Symbiose aus praktischer Nutzengenerierung sowie wissenschaftlichem Erkenntnisgewinn. Der Beitrag stellt den gewählten Forschungsansatz anhand konkreter Beispiele vor. Darauf basierend werden Empfehlungen und Herausforderungen für eine gestaltungs- und praxisorientierte Forschung diskutiert.

Schlüsselwörter Design Science Research · Anwendungsorientierter Forschungsansatz $\cdot$ Praxisprojekte $\cdot$ Referenzmodellierung $\cdot$ Enhanced Telecom Operations Map

C. Czarnecki $(\bowtie)$

Hochschule Hamm-Lippstadt, Marker Allee 76-78, 59063 Hamm, Deutschland

E-Mail: christian.czarnecki@hshl.de

C. Dietze

MEA \& Turkey, Detecon Consulting FZ-LLC, Hazaa bin Zayed the 1st Street, 52612, Abu Dhabi, Vereinigte Arabische Emirate

E-Mail: christian.dietze@detecon.com 


\title{
Design Science Research using the example of Reference Modeling in the Telecommunications Industry
}

\begin{abstract}
Using the example of the telecommunications industry, the article shows a concrete form of application-oriented research that provides findings for both practice and science. The research subjects are the reference models of the industry committee TM Forum, which are used by many telecommunications companies to transform their structures and systems. The long-term research activity in the further development and application of these reference models is described. A consistently design-oriented research approach is pursued. The interplay of continuous further development in cooperation with an industry committee and its use in a wide range of practical projects leads to a successful symbiosis of practical benefit generation and scientific findings. The article presents the chosen research approach using concrete examples. Based on this, recommendations and challenges for design-oriented and practice-oriented research are discussed.
\end{abstract}

Keywords Design science research - Application-oriented research · Practical projects $\cdot$ Reference modeling $\cdot$ Enhanced Telecom Operations Map

\section{Bedarf an gestaltungs- und anwendungsorientierter Forschung}

Die anwendungsorientierte Gestaltung und Weiterentwicklung von (IT-)Artefakten ist ein wichtiges Ziel der Wirtschaftsinformatik (Jung and Lehrer 2017), wodurch ein Austausch der Forschung mit der Praxis naheliegend ist. Grundsätzlich wird die Entwicklung neuer Artefakte durch die gestaltungsorientierte Forschung (Design Science Research) behandelt (z. B. Hevner et al. 2004). In diesem Beitrag wird am konkreten Beispiel der Telekommunikationsindustrie (TK-Industrie) dargestellt, wie Forschung für die Praxis erfolgen kann. Die TK-Industrie befindet sich seit Jahrzehnten in einem enormen Wandel (z. B. Picot 2006; Bub et al. 2011; Plunkett 2018). Der damit verbundene Transformationsbedarf bietet umfangreiche Forschungspotentiale an und ist somit Gegenstand vielfältiger Publikationen (z. B. Czarnecki et al. 2012; Czarnecki and Dietze 2017a).

Aus Praxissicht besteht ein Bedarf an domänenspezifischen Referenzlösungen, welcher durch die Industrieorganisation TM Forum adressiert wird (TM Forum 2015a). In diesem Zusammenhang haben die beiden Autoren seit über 10 Jahren die Weiterentwicklung und Anwendung anerkannter Referenzmodelle im Zusammenspiel mit der Praxis erforscht und in unterschiedlichen Publikationen veröffentlicht (z. B. Czarnecki et al. 2010, 2013; Czarnecki 2013; Czarnecki and Dietze 2017a, b). Ziel des vorliegenden Artikels ist die übergreifende Darstellung des gewählten Forschungsansatzes als Diskussionsbeitrag zur Frage, wie Forschung für die Praxis erfolgreich gestaltet werden kann. Insofern wird im Folgenden von den konkreten Forschungsergebnissen abstrahiert. Diese werden ausschließlich zusammengefasst und beispielhaft dargestellt, um den gewählten Forschungsansatz zu verdeutlichen. Dabei werden grundsätzliche Empfehlungen und Herausforderungen für gestaltungsund anwendungsorientierte Forschung abgeleitet. 
Der Beitrag folgt selber dem gestaltungsorientierten Forschungsparadigma. Die Problemdomäne TK-Industrie und die methodische Basis der Referenzmodellierung werden in Abschn. 2 diskutiert. Danach wird der gewählte Forschungsansatz vorgestellt, wobei zwischen Designmethode (vgl. Abschn. 3.1) und gestaltetem Artefakt, also dem konkreten Referenzmodell (vgl. Abschn. 3.2), unterschieden wird. Die Anwendung in Praxisprojekten wird in Abschn. 3.3 diskutiert. Sie stellt aus Forschungssicht die Evaluation und aus Praxissicht die Nutzengenerierung dar. In Abschn. 3.4 folgt ein Ausblick auf die aktuelle Weiterentwicklung der Referenzmodelle im TM Forum. Aus genereller Sicht werden in Abschn. 4 konkrete Empfehlungen und Herausforderungen abgeleitet.

\section{Referenzmodellierung in der Telekommunikationsindustrie}

\subsection{Herausforderungen der Telekommunikationsindustrie}

Die Telekommunikationsindustrie (TK-Industrie) durchläuft derzeit einen erheblichen Wandel, der sich auf Strategie, Struktur und Technologie der Akteure entlang der gesamten Wertschöpfungskette auswirkt (z.B. Grover and Saeed 2003; Picot 2006; Czarnecki and Dietze 2017a, b). Unter dem Stichwort Digitale Transformation werden aktuell fundamentale Veränderungen in unterschiedlichen Branchen beobachtet, die in vielen Fällen durch Informations- und Kommunikations-Technologie (IKT) ermöglicht werden (z. B. Bensberg and Buscher 2016). Gerade die TKIndustrie wird dabei als ein wichtiger Treiber der Digitalen Transformation angesehen. Mit einem globalen Jahresumsatz von rund 6,5 Billionen USD (Plunkett 2018) stellt sie einen wichtigen Teil des IKT-Sektors dar. Nichtsdestotrotz ist die TK-Industrie in der Gesamtbetrachtung ein stagnierender Markt, wobei die Entwicklung nach Übertragungstechniken unterschiedlich ausfällt. Die Gesamtzahl der Festnetzanschlüsse nimmt ab, und auch die Wachstumsrate der Mobilfunkanschlüsse sinkt, während mobile Breitbandanschlüsse (z.B. LTE) ein enormes Wachstum aufweisen (ITU 2015a; Plunkett 2018). Diese veränderten Nutzungsgewohnheiten gehen mit einem allgemeinen Preisverfall für die meisten Kommunikationsdienste einher (ITU 2015b; Plunkett 2018). Einerseits erfordert die steigende Nachfrage nach hohen Übertragungsbandbreiten umfangreiche Investitionen in die Netzinfrastruktur, andererseits wird die rein technische Übertragung zur Selbstverständlichkeit. Over-the-Top (OTT)-Anbieter realisieren neue Kommunikationsdienste ohne eigene Netzinfrastruktur und schaffen einen neuen Wettbewerb für traditionelle TK-Unternehmen. Einige Dienste der OTT-Anbieter haben bereits gleichwertige Kommunikationsdienste ersetzt (bspw. WhatsApp). Während das reine Übertragungsgeschäft stagniert, besteht für TK-Unternehmen in vertikalen Märkten ein Wachstumspotenzial, z. B. in den Bereichen Automobil, Banken, Gesundheitswesen, Versicherungen, Transport und Logistik sowie Smart Home (Czarnecki and Dietze 2017a).

Die technische Übertragung wird zu einem kleinen Teil der gesamten Wertschöpfungskette, die nun mit neuen Akteuren, Fusionen und Übernahmen konfrontiert ist (Wulf and Zarnekow 2011). Die Konvergenz von Kommunikationsdiensten, mobile Endgeräte sowie virtuelle Geschäftsmodelle (Pousttchi and Hufenbach 2011) wirken 
sich auf die Wertschöpfung aus. Diese entwickelt sich zu einem Wertschöpfungsnetz, das aus Netzbetreibern, Softwareanbietern, Finanzintermediären, Inhalteanbietern, Portalen und Wiederverkäufern besteht. Neue Produkte und Dienstleistungen sind stark mit den veränderten Marktbedingungen und der Wertschöpfung verknüpft. So erzeugt ein Smartphone beispielsweise mehr als das 14-fache des Datenvolumens eines traditionellen Mobiltelefons (Verma and Verma 2014). TK-Unternehmen sind mit kontinuierlichen Innovationen (Picot 2006) und kürzeren Produktentwicklungszyklen (Bruce et al. 2008) konfrontiert. Sowohl auf der Anwendungs- als auch auf der Technologieseite wirkt sich die Konvergenz auf die Produktionsstruktur aus. So ist bspw. die starke Verbindung zwischen dem Telefonnetz und dem zugeordneten Telefondienst nicht mehr gültig. IP-Dienste realisieren heute Telefonie unabhängig vom Übertragungsnetz.

Die damit verbundenen Herausforderungen für die TK-Industrie sind Gegenstand vielfältiger Forschungsarbeiten, wie bspw. Wertschöpfung und Marktteilnehmer (Grover and Saeed 2003; Pousttchi and Hufenbach 2011; Wulf and Zarnekow 2011), Strukturen und Prozesse (Bruce et al. 2008; Czarnecki et al. 2013; Czarnecki and Dietze 2017b) sowie verschiedene funktionale oder technische Lösungen. In der Praxis passen TK-Unternehmen ihre Strategien, Strukturen und Technologien aufgrund der beschriebenen Branchenänderungen kontinuierlich an. Daher besteht ein Bedarf an domänenspezifischen Referenzlösungen, welcher durch die Industrieorganisation TM Forum adressiert wird. Insofern bietet die TK-Industrie ein relevantes Beispiel für das Zusammenspiel zwischen praxisorientierter Forschung und konkreter Umsetzung in der Praxis.

\subsection{Referenzmodelle des TM Forums}

Die Industrieorganisation TM Forum bietet konkrete Referenzmodelle für die TKIndustrie an (Kelly 2003; Czarnecki and Dietze 2017b). Das TM Forum ist eine international anerkannte Organisation, die 1988 von mehreren etablierten TKUnternehmen gegründet wurde. Heute zählt es mehr als 850 Mitgliedsunternehmen, die von Kommunikationsdienstleistern über Softwareanbieter bis hin zu Systemintegratoren reichen. Mit Arbeitsgruppen, Workshops und Konferenzen bietet es ein Ökosystem für gemeinsame Entwicklungs- und Forschungsaktivitäten. Die Mitgliedschaft steht Unternehmen und Forschungseinrichtungen offen. Die Mitglieder können sich in den Arbeitsgruppen einbringen und alle bereitgestellten Inhalte in der Praxis nutzen. ${ }^{1}$ Das TM Forum stellt die folgenden drei Referenzmodelle für Prozesse, Daten und Anwendungssysteme bereit (TM Forum 2015a):

1. enhanced Telecom Operations Map (eTOM) bietet Prozessdefinitionen und Prozessabläufe auf Basis einer hierarchischen Struktur (TM Forum 2015b).

2. Shared Information/Data Model (SID) bietet eine Datenstruktur und detaillierte Entity-Relationship-Modelle (ERM) (TM Forum 2015c).

\footnotetext{
1 Insofern gilt die Creative Commons Lizenz nicht für die hier dargestellten Inhalte des TM Forums (z. B. eTOM-Prozesse). Für die anzuwendenden Nutzungsbedingungen siehe www.tmforum.org.
} 
3. Telecom Application Map (TAM) konzentriert sich auf Funktionalitäten für Anwendungssysteme (TM Forum 2015d).

Im Hinblick auf die diskutierten Herausforderungen und Anforderungen unterstützen die Referenzmodelle des TM Forums die TK-Unternehmen durch kundenorientierte Prozesse sowie konsequente Entkopplung zwischen Marktanforderungen und technischen Realisierungen (Czarnecki and Dietze 2017b). Die vorgeschlagenen Modelle unterscheiden zwischen Produkt, Dienstleistung und Ressource. Sie werden mit klaren Prozessdomänen kombiniert, und daher helfen diese Referenzmodelle, die Flexibilität zu erhöhen und die technische Komplexität zu verallgemeinern. Darüber hinaus werden sie von branchenspezifischen Standardsoftwaresystemen angewendet.

Alle drei Referenzmodelle werden in Arbeitsgruppen des TM Forums kontinuierlich aktualisiert. Sie spiegeln einen Konsens innerhalb der TK-Industrie wider. Darüber hinaus hat die International Telecommunications Union (ITU) Teile der Referenzmodelle des TM Forums als De-facto-Standard bestätigt. Die beiden Autoren sind seit über 10 Jahren aktiv im TM Forum an der Weiterentwicklung des Referenzmodells eTOM beteiligt, was der weitere Fokus dieses Beitrags ist.

\section{Forschung für die Praxis am Beispiel der Telekommunikationsindustrie}

\subsection{Ablauf und Vorgehen}

Grundsätzlich sind Referenzmodelle ein gängiges Forschungsartefakt der Wirtschaftsinformatik (z.B. Fettke et al. 2006; Farhadi 2019). Während ein konkretes Modell eine Lösung für eine klar definierte Situation liefert, stellt ein Referenzmodell einen Bezugspunkt für eine ganze Reihe von Situationen dar (Fettke and Loos 2007). Gegenstand dieses Artikels ist die Erforschung und Weiterentwicklung des Referenzprozessmodells eTOM. Damit liegt ein domänenspezifisches Referenzmodell vor, das in der Praxis anerkannt und seit Jahren Gegenstand der anwendungsorientierten Forschung ist (z.B. Kelly 2003; Czarnecki et al. 2013; Czarnecki and Dietze 2017b).

Die von den Autoren durchgeführten Arbeiten (z. B. Czarnecki et al. 2012; Czarnecki and Dietze 2017a, b) sind dabei der gestaltungsorientierten Forschung zuzuordnen (Hevner et al. 2004). Durch das TM Forum ist eine enge Verknüpfung mit der Praxis und damit eine hohe Relevanz gewährleistet. Das Referenzprozessmodell eTOM wird in einer Arbeitsgruppe des TM Forums gemeinsam von den Mitgliedern gestaltet und weiterentwickelt. Dabei stellt es in Bezug auf Prozesse in der TK-Industrie einen Konsens sowie eine Empfehlung der beteiligten Unternehmen dar. Aus Praxissicht ist die Anwendung von eTOM in einem konkreten Unternehmenskontext das originäre Ziel. Aus den Erfahrungen bei der Anwendung werden allgemeine Lösungsvorschläge als Weiterentwicklung von eTOM generiert. Genau in diesem Zusammenspiel aus Weiterentwicklung des bestehenden Referenzmodells und Anwendung in Praxisprojekten ist der hier vorgestellte Forschungsansatz angesiedelt (vgl. Abb. 1). 


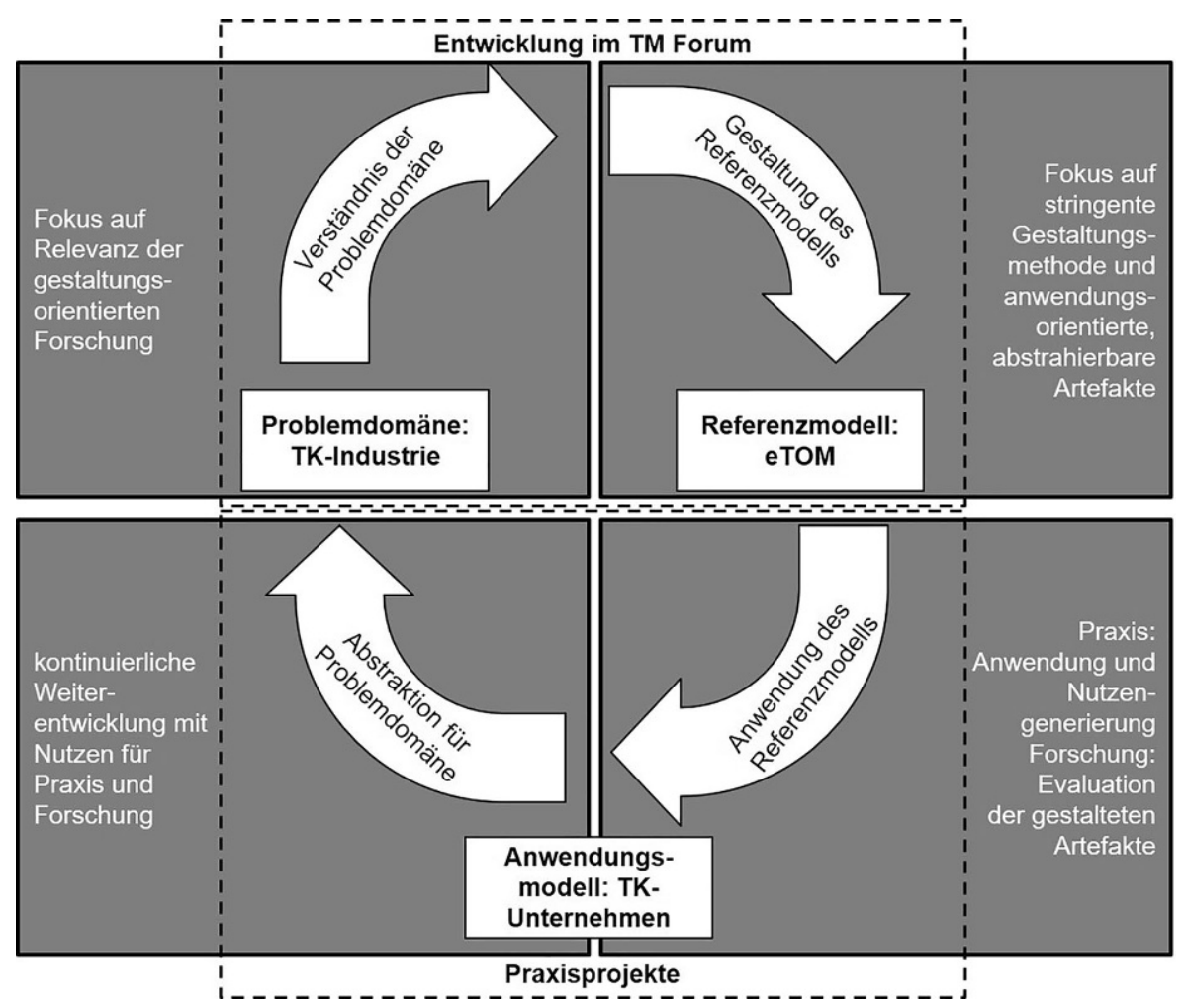

Abb. 1 Zusammenspiel zwischen Forschung und Praxis

Auch wenn der tatsächliche praktische Nutzen in der Anwendung in Praxisprojekten besteht, wird auch in der anwendungsorientierten Forschung die reine Anwendung bestehenden Wissens nicht als Erkenntnisgewinn angesehen, sondern es wird eine Neuartigkeit durch (Weiter-)Entwicklung des Artefakts oder Übertragung auf andere Problemdomänen erwartet (Gregor and Hevner 2013). Dieser für die anwendungsorientierte Forschung typische Konflikt zwischen praktischer Relevanz und wissenschaftlicher Rigorosität wird von den Autoren durch die stetige Abstraktion von der konkreten Anwendung und durch die Weiterentwicklung des Referenzmodells eTOM adressiert. Somit ist der Erkenntnisgewinn dem gestaltungsorientierten Forschungsziel der Entwicklung neuer Lösungen für einen bekannten Problembereich zuzuordnen (Gregor and Hevner 2013). Die Darstellung der Forschungsergebnisse erfolgt dabei gemäß der folgenden Struktur: (1) Verständnis der Problemdomäne, (2) Vorgehen beim Design, (3) Designartefakt als Weiterentwicklung von eTOM, (4) Evaluation anhand von Praxisprojekten und (5) Diskussion und Ausblick. Außerdem wurden einzelne Elemente durch empirische Untersuchungen untermauert. Neben einem konkreten Erkenntnisgewinn für die Problemdomäne der TK-Industrie wurden auch Beiträge zu Methoden des Prozessmanagements und der Unternehmensmodellierung geleistet. 


\subsection{Darstellung des Artefakts}

Das konkrete Forschungsartefakt wird im Folgenden beispielhaft und zusammengefasst in Anlehnung an Czarnecki und Dietze (2017b) dargestellt, um den gewählten Forschungsansatz zu verdeutlichen. Die Kernelemente von eTOM können in (1) Prozessdefinitionen, die eine Sammlung und Kategorisierung von Geschäftsprozessen speziell für TK-Unternehmen ermöglichen (Kelly 2003), und (2) Referenzprozessabläufe, die eine Reihenfolge für diese Prozessdefinitionen vorgeben (Czarnecki et al. 2013), strukturiert werden (siehe Abb. 2). Beide sind hierarchisch auf verschiedenen Detaillierungsstufen ausgestaltet (Czarnecki and Dietze 2017a, b). Jeder Teil von eTOM enthält spezifische Design-Artefakte, bspw. wird auf Ebene 0-1 ein Prozess-Framework vorgeschlagen und auf Ebene 3 detaillierte Referenz-Prozessabläufe bereitgestellt. Diese Design-Artefakte sind miteinander verknüpft, bspw. werden die Prozessdefinitionen der Stufe 3 nach Stufe 2 kategorisiert und als Input für die detaillierten Referenzprozessabläufe auf Stufe 3 verwendet. Darüber hinaus enthält eTOM verschiedene methodische Empfehlungen, eine allgemeine Konzeptbeschreibung und Anwendungshinweise, die spezifische Implementierungen vorstellen. eTOM besteht daher aus vielen verschiedenen Dokumenten, einer XMLbasierten Darstellung sowie Modelldateien in verschiedenen Formaten.

Die eTOM-Prozessdefinition unterscheidet auf höchster Ebene die folgenden drei Prozessgruppen (TM Forum 2015c):

1. Operations enthält alle Prozesse, die erforderlich sind, um ein TK-Unternehmen unter der Annahme bestehender Infrastruktur und Produkte zu betreiben. Diese Prozesse umfassen z. B. Vertrieb, Kundenservice, Störungen und Abrechnung.

2. Strategie, Infrastruktur und Produkte (SIP) umfasst alle anderen domänenspezifischen Prozesse, die für ein TK-Unternehmen notwendig sind, d.h. die Planung und Implementierung seiner Infrastruktur und Produkte von der Strategieentwicklung bis zur technischen Umsetzung.

3. Enterprise Management bietet allgemeine Unterstützungsprozesse, z. B. Personalwesen, Finanzen und Kommunikation.

Die drei Prozessgruppen Operations, SIP und Enterprise Management werden mit den sechs horizontalen Einheiten aus dem eTOM-Framework (Level 0-1) kombiniert. Basierend auf dieser Struktur werden Prozessdetaillierungen vorgenommen (vgl. linker Teil von Abb. 2). Die Prozessdefinition von eTOM ist daher eine hierarchische Sammlung von Geschäftsprozessen. Für ein konkretes Prozessdesign kann diese Sammlung sowohl als einheitliche Terminologie als auch als Checkliste zur Identifizierung aller relevanten Prozesse verwendet werden. Die in den ersten eTOMPublikationen (d.h. GB921-D und GB921-DX) vorgeschlagenen Prozessdefinitionen enthalten jedoch keine Referenz für einen Prozessablauf, d.h. sie geben keine Aussage über die Reihenfolge. Im Rahmen der kontinuierlichen Weiterentwicklung von eTOM wurden Referenzprozessabläufe entwickelt (Czarnecki et al. 2013). Die Referenzprozessabläufe werden als offizielle Erweiterung zu eTOM in GB921-E veröffentlicht.

Die Referenzprozessabläufe basieren auf den folgenden Bereichen, die eine durchgängige Perspektive auf die Prozessabläufe bieten: 


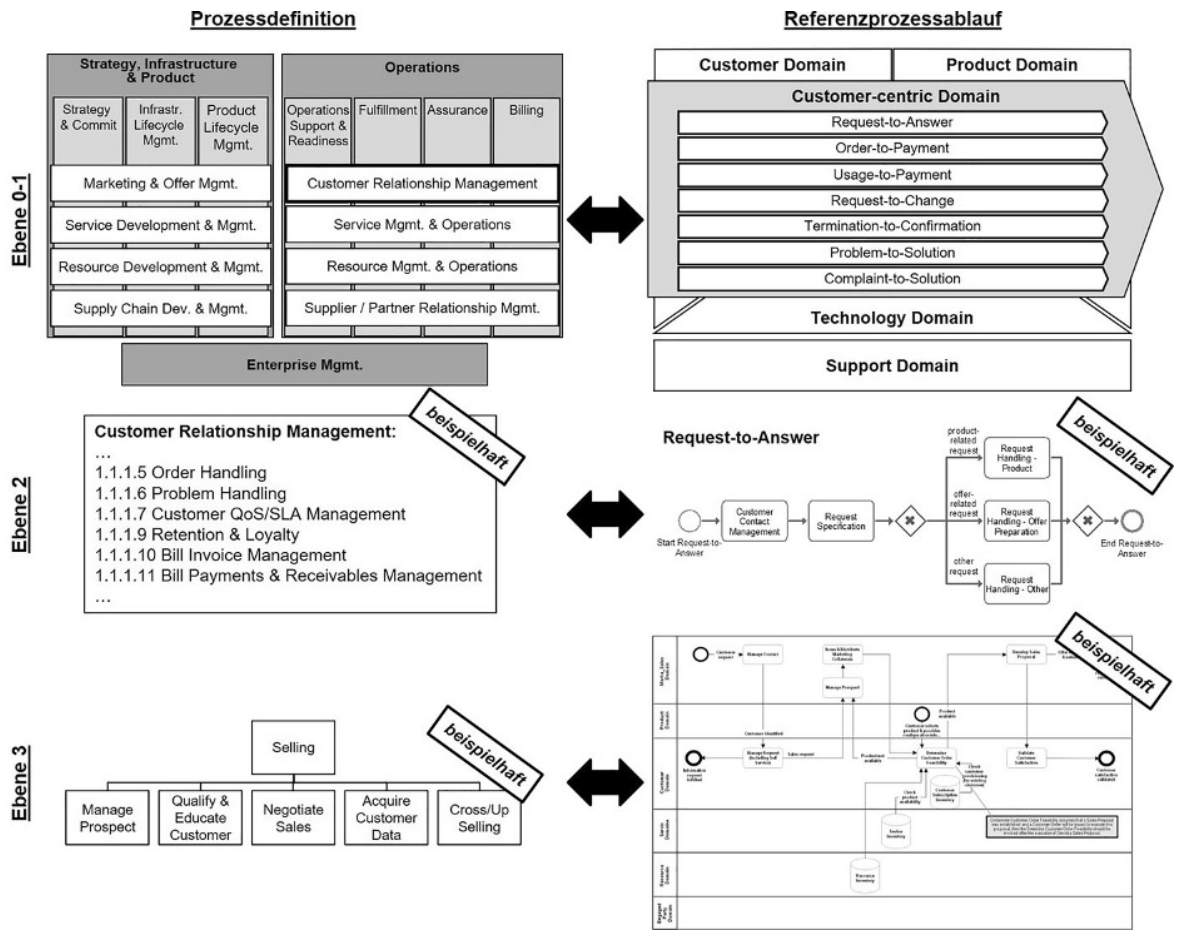

Abb. 2 Struktur des eTOM-Artefakts (in Anlehnung an Czarnecki and Dietze 2017b; die einzelnen Inhalte sind Gegenstand von eTOM und damit urheberrechtlich durch das TM Forum geschützt, bzgl. Nutzungsbedigungen siehe www.tmforum.org)

- Die Kundenzentrierte Domäne enthält alle Interaktionen, die direkt vom Kunden initiiert werden.

- Die Technologiedomäne umfasst die Realisierung und den Betrieb von Kommunikationsdiensten und Netzwerkressourcen.

- Die Produktdomäne reicht von der Produktentwicklung bis zur Produkteliminierung.

- Die Kundendomäne umfasst Interaktionen, die mit Kunden zu tun haben, aber nicht direkt vom Kunden initiiert werden (z. B. Marketingkampagnen).

- Die Supportdomäne umfasst allgemeine Unterstützungsprozesse (z.B. Finanzoder Personalmanagement).

Für jede Domäne werden Ende-zu-Ende-Prozessabläufe definiert, die wiederum gemäß der eTOM-Hierarchie detailliert werden. Mit dieser Zuordnung wird die Sequenz in die Prozessdefinition aufgenommen. Darüber hinaus werden die Referenzprozessabläufe auf verschiedenen Ebenen entlang der eTOM-Hierarchie definiert (vgl. rechter Teil von Abb. 2). Die kundenorientierte Domäne enthält bspw. den Ende-zu-Ende-Prozessablauf Anfrage-bis-Auskunft, der alle Prozessschritte vom Kundenkontakt bis zur Beantwortung der Anfrage abdeckt. 
Tab. 1 Beispielhafte Praxisprojekte (in Anlehnung an Czarnecki and Dietze 2017b)

\begin{tabular}{|c|c|c|c|}
\hline Gegenstand & Vorgehen & Artefakt & Evaluation \\
\hline \multicolumn{4}{|c|}{ Praxisprojekt 1: Verbesserung des Problembehebungsprozesses } \\
\hline $\begin{array}{l}\text { - Etabliertes TK- } \\
\text { Unternehmen im } \\
\text { Mittleren Osten } \\
\text { (ehemaliger } \\
\text { Monopolist) } \\
\text { - Verstärkter } \\
\text { Wettbewerb } \\
\text { bei gleichzeitig } \\
\text { sinkender Kun- } \\
\text { denzufriedenheit } \\
\text { - Verbesserung des } \\
\text { Problembehe- } \\
\text { bungsprozesses } \\
\text { als Bestandteil } \\
\text { eines umfangrei- } \\
\text { chen Transforma- } \\
\text { tionsprogramms }\end{array}$ & $\begin{array}{l}\text { - Analyse der Ist-Pro- } \\
\text { zesse basierend auf } \\
\text { eTOM (Ebene 0-1) } \\
\text { - Durchführung von } \\
\text { Interviews zum Ver- } \\
\text { ständnis der aktuell } \\
\text { implementierten } \\
\text { Prozesse } \\
\text { - Gestaltung der Soll- } \\
\text { Prozesse basierend } \\
\text { auf den eTOM-Refe- } \\
\text { renzprozessabläufen } \\
\text { (Ebene 3) } \\
\text { - Zuordnung auf beste- } \\
\text { hende Organisations- } \\
\text { struktur } \\
\text { - Anpassung an spezi- } \\
\text { fische Anforderungen } \\
\text { basierend auf Work- } \\
\text { shops }\end{array}$ & $\begin{array}{l}\text { - Detaillierte Sollprozesse } \\
\text { als konkretes Anwen- } \\
\text { dungsmodell für das TK- } \\
\text { Unternehmen } \\
\text { - Insgesamt } 35 \text { Prozess- } \\
\text { diagramme basierend auf } \\
\text { eTOM }\end{array}$ & $\begin{array}{l}\text { - Implementierung } \\
\text { der Soll- } \\
\text { Prozesse } \\
\text { innerhalb von } \\
8 \text { Monaten } \\
\text { - Konkrete Leis- } \\
\text { tungsverbesse- } \\
\text { rungen wurden } \\
\text { nach der Im- } \\
\text { plementierung } \\
\text { gemessen, z. B. } \\
\text { Reduktion der } \\
\text { prozessbezo- } \\
\text { genen Arbeits- } \\
\text { zeiten um 39\%, } \\
\text { Reduktion der } \\
\text { gemeldeten } \\
\text { Störungen um } \\
23 \%\end{array}$ \\
\hline \multicolumn{4}{|c|}{ Praxisprojekt 2: Implementierung eines Network Operations Center } \\
\hline $\begin{array}{l}\text { - Afrikanisches } \\
\text { TK-Unterneh- } \\
\text { men (Festnetz } \\
\text { und Mobilfunk) } \\
\text { - Gründung eines } \\
\text { übergreifenden } \\
\text { Network Ope- } \\
\text { rations Center } \\
\text { (NOC) } \\
\text { - Gestaltung von } \\
\text { Zielprozessen für } \\
\text { das neue NOC }\end{array}$ & $\begin{array}{l}\text { - Durchführung in drei } \\
\text { Phasen } \\
\text { - Phase 1: Analyse der } \\
\text { bestehenden Prozesse } \\
\text { - Phase 2: Gestaltung } \\
\text { eines Ordnungsrah- } \\
\text { mens für die Zielpro- } \\
\text { zesse } \\
\text { - Phase 3: Entwurf } \\
\text { detaillierter Zielpro- } \\
\text { zesse } \\
\text { - Nutzung der Tech- } \\
\text { nologiedomäne von } \\
\text { eTOM als Referenz } \\
\text { in allen drei Phasen }\end{array}$ & $\begin{array}{l}\text { - Komplettes Prozessde- } \\
\text { sign Ebene } 0 \text { bis } 5 \text { als } \\
\text { Zielprozesse für das neue } \\
\text { NOC } \\
\text { - Anpassung der eTOM- } \\
\text { Referenzabläufe basie- } \\
\text { rend auf den spezifischen } \\
\text { Anforderungen des TK- } \\
\text { Unternehmens } \\
\text { - Zuordnung von operati- } \\
\text { ven Verantwortlichkeiten } \\
\text { und Leistungskennzahlen }\end{array}$ & $\begin{array}{l}\text { - Abstimmung al- } \\
\text { ler Zielprozesse } \\
\text { mit den ver- } \\
\text { antwortlichen } \\
\text { Personen } \\
\text { - Unabhängige } \\
\text { Zertifizierung } \\
\text { aller Zielpro- } \\
\text { zesse durch das } \\
\text { TM Forum } \\
\text { - Bestätigung der } \\
\text { Qualität und } \\
\text { Konformität } \\
\text { durch das TM } \\
\text { Forum }\end{array}$ \\
\hline
\end{tabular}

\subsection{Anwendung in Praxisprojekten}

Die konkrete Anwendung in Praxisprojekten ist ein wichtiger Bestandteil des hier dargestellten anwendungsorientierten Forschungsansatzes. Insofern sind die beiden Autoren kontinuierlich an der Anwendung in Praxisprojekten beteiligt. Aus der Perspektive der Praxis ist diese Anwendung der eigentliche Zweck des Referenzmodells eTOM, und auch erst dadurch entsteht ein wahrgenommener Nutzen. Aus der Perspektive der Wissenschaft sind die Praxisprojekte als Evaluation anzusehen, und damit unverzichtbarer Bestandteil gestaltungsorientierter Forschung. Für beide Perspektiven ist die Rückkopplung zwischen Referenzmodell und Anwendung wichtig, um eine kontinuierliche Weiterentwicklung zu ermöglichen. 
Grundsätzlich sollte ein Referenzmodell für eine bestimmte Problemdomäne generalisierbar sein, und seine Anwendung ist von seiner Entwicklung entkoppelt (vom Brocke 2015). Daher kann seine allgemeine Gültigkeit nicht nachgewiesen werden, wird aber mit jeder Anwendung wahrscheinlicher. Die Anwendung eines Referenzmodells kann durch Fallstudien veranschaulicht werden, die als valide Evaluation von Designartefakten angesehen werden (Hevner et al. 2004). Für eine detaillierte Diskussion der Evaluation der in diesem Beitrag beispielhaft vorgestellten Artefakte siehe z. B. Czarnecki (2013).

Bei der Anwendung des Referenzmodells eTOM steht die Anpassung an konkrete Spezifika des Praxisprojektes im Vordergrund. Diese werden bspw. von organisatorischen Verantwortlichkeiten und implementierten Anwendungssystemen abgeleitet. Häufig sind dabei historisch gewachsene Strukturen und Systeme als begrenzende Faktoren zu beachten. Während eTOM genau von diesen spezifischen Besonderheiten abstrahiert, entsteht der Nutzen im konkreten Praxiskontext erst durch die jeweilige Anpassung. Meist erfordern diese Anpassungen iterative Abstimmungsrunden mit den zukünftigen Modellnutzern.

Im Sinne der gestaltungsorientierten Forschung stellt das Praxisprojekt die Validierung der entwickelten Artefakte dar (Rigorosität des Gestaltungsprozesses) und liefert Einblicke in die konkrete Ausgestaltung der Problemdomäne (Relevanz der Artefakte). Tab. 1 fasst beispielhaft zwei konkrete Praxisprojekte zusammen (in Anlehnung an Czarnecki and Dietze 2017a, b; Czarnecki 2018). Grundsätzlich besteht die Herausforderung bei der Bewertung der Praxisprojekte, zwischen (1) spezifischen Besonderheiten des Projektes ohne jegliche weitere Relevanz und (2) abstrahierbaren Erkenntnissen zur Weiterentwicklung von eTOM zu unterscheiden. So wäre bspw. eine aufgrund der Organisation erforderliche Trennung zwischen Störungen im Festnetz und Mobilfunk der ersten Kategorie zuzuordnen, während Schnittstellen zu Inhalteanbietern als Erweiterung des Entstörungsprozesses der zweiten Kategorie angehören.

\subsection{Aktuelle Weiterentwicklung im TM Forum}

Ein weiterer wichtiger Grundgedanke des hier vorgestellten anwendungsorientierten Forschungsansatzes ist die kontinuierliche Weiterentwicklung im Rahmen des TM Forums. Im Folgenden wird beispielhaft die aktuelle Entwicklung eines digitalen Reifegradmodells als Erweiterung der bestehenden Referenzmodelle vorgestellt. Dazu wurde im TM Forum eine neue Arbeitsgruppe gegründet, an der auch die Autoren beteiligt sind. Ziel ist es, die Digitale Transformation für TK-Unternehmen anhand von vordefinierten Kriterien besser planen zu können, und die Umsetzung messbar zu machen. Die Arbeitsgruppe besteht aus TK-Unternehmen, Systemintegratoren und Unternehmensberatungen. Die Entwicklung hat einen starken Praxisfokus und basiert auf den Erfahrungen und Sichtweisen der involvierten Unternehmen. Als Ergebnis wird das TM Forum Digital Maturity Model (DMM) vorgeschlagen, das bereits in ersten Pilotprojekten eingesetzt wurde. Aus Sicht des TM Forums stellt das DMM das erste abgestimmte Reifegradmodell für die Planung und Umsetzung Digitaler Transformationen in der TK-Industrie dar. 


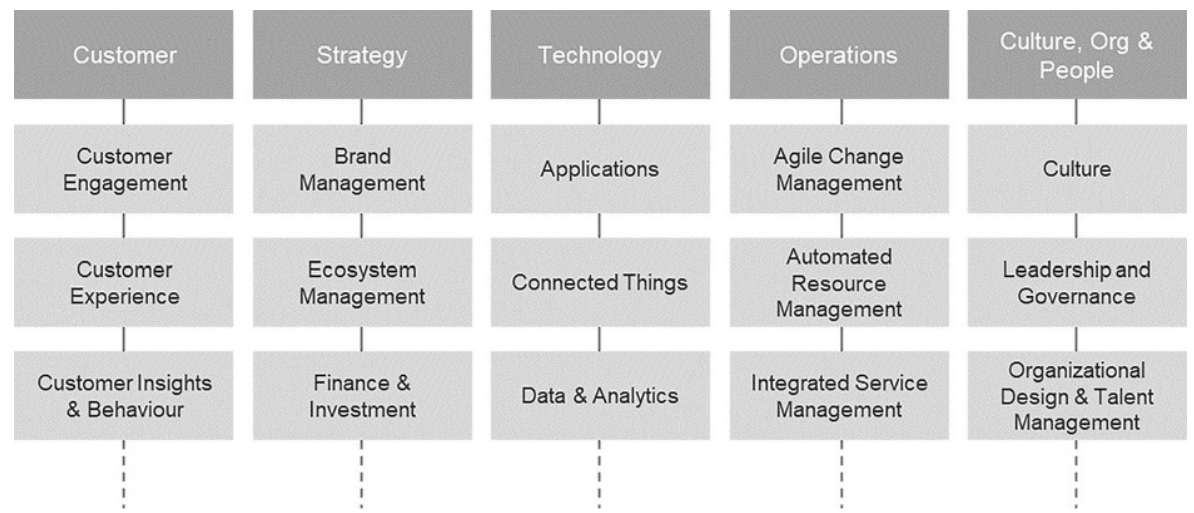

Abb. 3 Vereinfachte Darstellung des TM Forum Digital Maturity Models (DMM)

Bei der Entwicklung hat sich die Arbeitsgruppe an den folgenden Leitfragen orientiert: (1) Was bedeutet die Digitalisierung für das konkrete TK-Unternehmen? (2) Wie kann der Soll-Zustand in verschiedenen Dimensionen des TK-Unternehmens konkret definiert werden? (3) Wie hoch ist der digitale Reifegrad (Ist-Zustand) in diesen Dimensionen im Moment? (4) Durch welche Maßnahmen kann das TKUnternehmen transformiert werden, um vom Ist-Zustand in den Soll-Zustand zu gelangen?

Das zur Beantwortung dieser Fragen entwickelte DMM umfasst fünf Hauptdimensionen sowie zahlreiche Unterdimensionen (siehe Abb. 3). Den einzelnen Dimensionen sind ca. 175 Kriterien zugeordnet, anhand derer der aktuelle digitale Reifegrad in ausgewählten Bereichen des TK-Unternehmens gemessen und ein entsprechender Soll-Zustand definiert werden kann.

Für die praktische Anwendung des DMM haben die beiden Autoren ein Vorgehen entlang von sechs Phasen entwickelt:

- Phase 1: Auswahl der relevanten Geschäftsdimensionen

- Phase 2: Bewertung des aktuellen Reifegrades in den ausgewählten Geschäftsdimensionen

- Phase 3: Differenzanalyse zwischen bewertetem digitalen Reifegrad und digitalem Soll-Zustand

- Phase 4: Definition und Detaillierung konkreter Optimierungsmaßnahmen zur Schließung der identifizierten Lücken

- Phase 5: Priorisierung von Optimierungsmaßnahmen und Roadmap zur digitalen Transformation

- Phase 6: Projektmanagement und Überwachung der Umsetzung von Optimierungsmaßnahmen

Aktuell wurden erste erfolgreiche Praxisprojekte zur Anwendung des DMM durchgeführt. Diese stellen den Startpunkt für zukünftige Weiterentwicklungen im TM Forum sowie wissenschaftliche Erkenntnisgewinne dar. 


\section{Diskussion des vorgestellten Forschungsansatzes}

Der dargestellte Forschungsansatz zeigt am Beispiel der TK-Industrie, wie im Zusammenspiel aus Wissenschaft und Praxis iterativ ein anwendungsorientierter Erkenntnisgewinn erzielt werden kann. Dabei haben sich folgende Rahmenbedingungen als vorteilhaft gezeigt:

- Zusammenarbeit mit einem Industriegremium vereinfacht die Abstraktion von konkreten Problemstellungen.

- Fokussierung auf ein anerkanntes und bestehendes Referenzmodell schafft den anwendungsorientierten Rahmen für einen wissenschaftlichen Erkenntnisgewinn.

- Kontinuierliche Anwendung in Praxisprojekten ermöglicht die iterative Validierung und Weiterentwicklung.

- Gemeinsame Veröffentlichungen in Forschung und Praxis fördern das gegenseitige Verständnis und damit auch den Austausch.

Die folgenden Herausforderungen können zusammenfassend festgehalten werden:

- Wahrnehmung der Relevanz der Problemdomäne variiert teilweise in Forschung und Praxis. Während in der Praxis die TK-Industrie mit den verbundenen Herausforderungen als eigenständiges Problemfeld unstrittig ist, ist ein industriespezifischer Fokus in der Wissenschaft durchaus erklärungsbedürftig.

- Balance zwischen Methode und Inhalt kann schwierig sein, da aus Praxissicht ein stärkerer Fokus auf den Inhalten liegt, während eine methodische Fundierung aus Forschungssicht unerlässlich ist. Eine zielgruppenspezifische Darstellung der Forschungsergebnisse kann dabei nützlich sein.

- Wissenschaftstheorie ist nur von einseitigem Interesse. Aus Forschungssicht ist die wissenschaftstheoretische Fundierung von existentieller Bedeutung. Aus Praxissicht wird die Bedeutung meist gering eingeschätzt.

- Darstellung von kontextspezifischen Anpassungen kann zu Missverständnissen führen. Nicht alle Gestaltungsentscheidungen der Praxisprojekte folgen einem objektiv nachvollziehbaren Vorgehen, wie es in einem wissenschaftlichen Rahmen gewünscht wäre. Trotzdem stellen die Praxisprojekte einen wichtigen Bestandteil sowohl aus Sicht der Relevanz als auch der Rigorosität dar.

- Dokumentation und Zugänglichkeit der Forschungsergebnisse unterscheidet sich in Praxis und Forschung. Die Forschungsergebnisse sind für Mitglieder des Industriegremiums frei verfügbar (darüber hinaus jedoch nicht), was aus Praxissicht unkritisch ist, in der wissenschaftliche Community teilweise zu Diskussionen führt. Außerdem ist die Dokumentation von Praxisprojekten ausschließlich in stark zusammengefasster und meist anonymisierter Form möglich.

- Iterative Weiterentwicklung eines anerkannten Referenzmodells wird teilweise unterschiedlich wahrgenommen. Während Nutzen und Relevanz in der Praxis gerade durch die Nutzung eines bestehenden Referenzmodells entsteht, variiert die Bewertung aus wissenschaftlicher Sicht. So erfüllen bspw. historisch gewachsene Strukturen des Referenzmodelles nicht den Anspruch eines strikt objektiven Vorgehens. 
- Institutionalisierung des Forschungsansatzes ist innerhalb einer stark durch Drittmittel getrieben Forschungslandschaft schwierig. Aus Praxissicht stellt die Weiterentwicklung von eTOM - also die wissenschaftliche Forschung - eine Themenentwicklung dar, die von den Mitgliedsunternehmen durch gemeinsam erarbeitete Artefakte unterstützt wird. Grundsätzlich versteht sich das TM Forum als nichtgewinnorientierte Organisation.

Zusammenfassend stellt die unterschiedliche Bewertung von Inhalten und Methoden eine Herausforderung dar. Während in der Praxis die Nutzengenerierung in der Gestaltung konkreter Lösungsartefakte gesehen wird, also in diesem Fall bspw. konkrete Sollprozesse angepasst an spezifische Organisationsstrukturen und Anwendungssysteme, liegt der wissenschaftliche Erkenntnisgewinn gerade in der Abstraktion von diesen Spezifika. Auch die methodische Fundierung wird meist in der Praxis als weniger wichtig angesehen, während sie teilweise eine existentielle Bedeutung in der Forschung hat.

Die dargestellten Beispiele können dazu genutzt werden, die vielfältigen Ausprägungen anwendungsorientierter Forschung besser zu verstehen. Aus wissenschaftstheoretischer Sicht können auf dieser Basis gestaltungsorientierte Forschungsansätze weiter geschärft werden. Aus wissenschaftspolitischer Sicht wäre zu überlegen, inwiefern die dargestellte anwendungsorientierte Forschung durch bestehende Strukturen und Anreizsysteme unterstützt wird.

Aus konkreter Sicht des Forschungsgegenstands der TK-Industrie bietet gerade auch die Digitale Transformation vielfältige Ansatzpunkte, wobei das Digital Maturity Model (DMM) als Startpunkt für weitere Forschungsaktivitäten dienen kann.

Funding Open Access funding provided by Projekt DEAL.

Open Access Dieser Artikel wird unter der Creative Commons Namensnennung 4.0 International Lizenz veröffentlicht, welche die Nutzung, Vervielfältigung, Bearbeitung, Verbreitung und Wiedergabe in jeglichem Medium und Format erlaubt, sofern Sie den/die ursprünglichen Autor(en) und die Quelle ordnungsgemäß nennen, einen Link zur Creative Commons Lizenz beifügen und angeben, ob Änderungen vorgenommen wurden.

Die in diesem Artikel enthaltenen Bilder und sonstiges Drittmaterial unterliegen ebenfalls der genannten Creative Commons Lizenz, sofern sich aus der Abbildungslegende nichts anderes ergibt. Sofern das betreffende Material nicht unter der genannten Creative Commons Lizenz steht und die betreffende Handlung nicht nach gesetzlichen Vorschriften erlaubt ist, ist für die oben aufgeführten Weiterverwendungen des Materials die Einwilligung des jeweiligen Rechteinhabers einzuholen.

Weitere Details zur Lizenz entnehmen Sie bitte der Lizenzinformation auf http://creativecommons.org/ licenses/by/4.0/deed.de.

\section{Literatur}

Bensberg F, Buscher G (2016) Digitale Transformation und IT-Zukunftsthemen im Spiegel des Arbeitsmarkts für IT-Berater - Ergebnisse einer explorativen Stellenanzeigenanalyse. In: Tagungsband zur Multikonferenz Wirtschaftsinformatik (MKWI) 2016. Technische Universität, Ilmenau, S 1007-1018 vom Brocke J (2015) Referenzmodellierung: Gestaltung und Verteilung von Konstruktionsprozessen. Logos, Berlin 
Bruce G, Naughton B, Trew D et al (2008) Streamlining the telco production line. J Telecommun Manag $1: 15-32$

Bub U, Picot A, Krcmar H (2011) The Future of Telecommunications. Bus Inf Syst Eng 3:265-267. https:// doi.org/10.1007/s12599-011-0178-0

Czarnecki C (2013) Entwicklung einer referenzmodellbasierten Unternehmensarchitektur für die Telekommunikationsindustrie. Logos, Berlin

Czarnecki C (2018) Establishment of a Central Process Governance Organization Combined with Operational Process Improvements. In: vom Brocke J, Mendling J (Hrsg) Business Process Management Cases. Springer, Cham, S 57-76

Czarnecki C, Dietze C (2017a) Reference architecture for the telecommunications industry. Springer, Cham

Czarnecki C, Dietze C (2017b) Domain-specific reference modeling in the telecommunications industry. In: Maedche A, vom Brocke J, Hevner A (Hrsg) Designing the digital transformation. Springer, Cham, S 313-329

Czarnecki C, Winkelmann A, Spiliopoulou M (2010) Services in electronic telecommunication markets: a framework for planning the virtualization of processes. Electron Mark 20:197-207

Czarnecki C, Winkelmann A, Spiliopoulou M (2012) Transformation in Telecommunication-Analyse und Clustering von Real-life Projekten. In: Mattfeld DC, Robra-Bissantz S (Hrsg) Multi-Konferenz Wirtschaftsinformatik 2012. GITO, Braunschweig, S 985-998

Czarnecki C, Winkelmann A, Spiliopoulou M (2013) Referenzprozessabläufe für Telekommunikationsunternehmen: Eine Erweiterung des eTOM-Modells. Wirtschaftsinformatik 55:83-97. https://doi.org/ 10.1007/s11576-013-0351-9

Farhadi N (2019) Referenzmodell des Business-Ökosystems. In: Cross-Industry Ecosystems. Springer, Wiesbaden, S 23-43

Fettke P, Loos P (2007) Perspectives on reference modeling. In: Fettke P, Loos P (Hrsg) Reference modeling for business systems analysis. IGI Global, Hershey, London, S 1-21

Fettke P, Loos P, Zwicker J (2006) Business process reference models: survey and classification. In: Hutchison D, Kanade T, Kittler J et al (Hrsg) Business process management workshops. Springer, Berlin, Heidelberg, S 469-483

Forum TM (2015a) Frameworks release 15.0.0: release notes (RN354), version 15.0.0

Forum TM (2015b) Business process framwork (eTOM): concepts and principles (GB921 CP), version 15.0 .0

Forum TM (2015c) Information framwork (SID): concepts and principles (GB922), version 15.0.0

Forum TM (2015d) Application framwork (TAM): concepts and principles (GB929 CP), version 14.5.1

Gregor S, Hevner AR (2013) Positioning and presenting design science research for maximum impact. Mis Q 37:337-356

Grover V, Saeed K (2003) The telecommunication industry revisited. Commun ACM 46:119-125. https:// doi.org/10.1145/792704.792709

Hevner AR, March ST, Park J, Ram S (2004) Design science in information systems research. Mis Q 28:75-105

ITU (2015a) Key ICT indicators for developed and developing countries and the world

ITU (2015b) ICT facts and figures - the world in 2015

Jung R, Lehrer C (2017) Guidelines for education in business and information systems engineering at tertiary institutions. Bus Inf Syst Eng 59:189-203. https://doi.org/10.1007/s12599-017-0473-5

Kelly MB (2003) The telemanagement forum's enhanced Telecom operations map (eTOM). J Netw Syst Manag 11:109-119

Picot A (Hrsg) (2006) The Future of Telecommunications Industries. Springer, Berlin, Heidelberg

Plunkett JW (2018) Plunkett's Telecommunications Industry Almanac 2019. Plunkett Research, Houston

Pousttchi K, Hufenbach Y (2011) Wertschöpfung im Mobilfunkmarkt. Wirtschaftsinformatik 53:287-300. https://doi.org/10.1007/s11576-011-0287-x

Verma DC, Verma P (2014) Techniques for surviving the mobile data explosion

Wulf J, Zarnekow R (2011) Branchenübergreifender Wettbewerb in der Telekommunikation. Wirtschaftsinformatik 53:277-286. https://doi.org/10.1007/s11576-011-0289-8 